\title{
The Morphology of Narrow Gaps Modifies the Plasmonic Response
}

\author{
Rubén Esteban, ${ }^{* \dagger}$ Garikoitz Aguirregabiria, ${ }^{\dagger}$ Andrey G. Borisov, ${ }^{\ddagger}$ Yumin M. Wang, ${ }^{\perp}$ Peter Nordlander, ${ }^{\perp}$
} Garnett W. Bryant, ${ }^{\S}$ and Javier Aizpurua*, ${ }^{* \dagger}$

${ }^{\dagger}$ Centro de Física de Materiales, Centro Mixto CSIC-UPV/EHU and Donostia International Physics Center (DIPC), 20018 Donostia-San Sebastián, Spain

${ }^{\ddagger}$ Institut des Sciences Moléculaires d’Orsay, UMR 8214, CNRS-Université Paris-Sud, 91405 Orsay, France

${ }^{\perp}$ Department of Electrical and Computer Engineering, MS378, Laboratory of Nanophotonics, Rice University, Houston, Texas 77005, United States

${ }^{\S}$ Joint Quantum Institute and Quantum Measurement Division, National Institute of Standards and Technology, Gaithersburg, Maryland 20899, United States

\section{Supporting Information}

ABSTRACT: The optical response of a plasmonic gapantenna is mainly determined by the Coulomb interaction of the two constituent arms of the antenna. Using rigorous calculations supported by simple analytical models, we observe how the morphology of a nanometric gap separating two metallic rods dramatically modifies the plasmonic response. In the case of rounded terminations at the gap, a conventional set of bonding modes is found that red-shifts strongly with
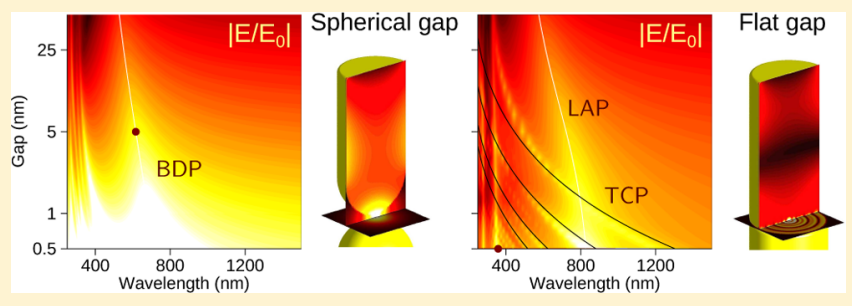
decreasing separation. However, in the case of flat surfaces, a distinctly different situation is found with the appearance of two sets of modes: (i) strongly radiating longitudinal antenna plasmons (LAPs), which exhibit a red-shift that saturates for very narrow gaps, and (ii) transverse cavity plasmons (TCPs) confined to the gap, which are weakly radiative and strongly dependent on the separation distance between the two arms. The two sets of modes can be independently tuned, providing detailed control of both the near- and far-field response of the antenna. We illustrate these properties also with an application to larger infrared gap-antennas made of polar materials such as SiC. Finally we use the quantum corrected model (QCM) to show that the morphology of the gap has a dramatic influence on the plasmonic response also for subnanometer gaps. This effect can be crucial for the correct interpretation of charge transfer processes in metallic cavities where quantum effects such as electron tunneling are important.

KEYWORDS: optical antennas, plasmonic gaps, cavity modes, antenna modes, quantum effects, quantum corrected model, plasmonic resonances, phononic resonances

$\mathrm{M}$ etallic particles can show a strong optical response due to the excitation of resonant plasmonic modes, making light manipulation possible in structures of dimensions comparable to or smaller than the wavelength. Typical effects are the efficient emission of radiation and the concentration of the incoming light energy in small volumes, thus justifying the characterization of these metallic structures as optical antennas. ${ }^{1,2}$ Modifications of the geometry, size, or materials ${ }^{3-6}$ affect the optical response. In particular, the resonant modes of optical antennas consisting of two metallic particles separated by a narrow gap show significant sensitivity to the properties of the gap. ${ }^{7-19}$

In an optical antenna, a change in geometry can simultaneously affect both the strength and wavelength of its resonances and modify both the far- and near-field response. It is usually a challenge, for example, to control the near field without affecting the far-field properties. In this theoretical work, we discuss how the coexistence ${ }^{20,21}$ of two different and mostly spectrally decoupled types of modes in flat-gap antennas, namely, transverse cavity modes ${ }^{22}$ and longitudinal antenna modes, ${ }^{15}$ allows for a more flexible tuning of far- and near-field properties compared to that of the conventional spherical-gap terminations ${ }^{13,17,23}$ The importance of the gap morphology ${ }^{24}$ is particularly pronounced for nanometer- and subnanometer-sized gaps, where strong interactions are present. We find a similar effect at larger separation distances for phononic antennas made of $\mathrm{SiC} .^{25}$ For gaps smaller than about half a nanometer, electron tunneling becomes a relevant process, affecting the optical response of metallic gapantennas. $^{26-31} \mathrm{We}$ investigate this effect using the quantum corrected model (QCM) ${ }^{32}$ and find that the effects of tunneling also depend on the structure of the antenna gap. The pronounced dependence of the plasmonic response on the gap morphology shows that gap engineering is a promising tool for precise control of both the far- and near-field properties of subwavelength antennas.

Received: October 29, 2014

Published: December 31, 2014 


\section{GAP-ANTENNA MORPHOLOGY}

To understand the effects of the gap morphology on the plasmonic response, we consider two types of nanoantennas, depicted in Figure 1. In both cases, the structure consists of two

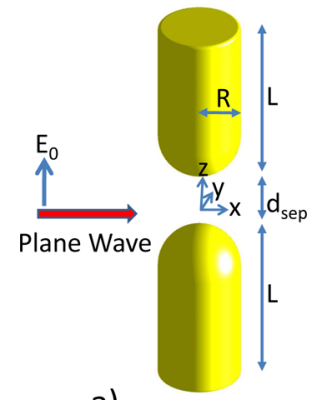

a)

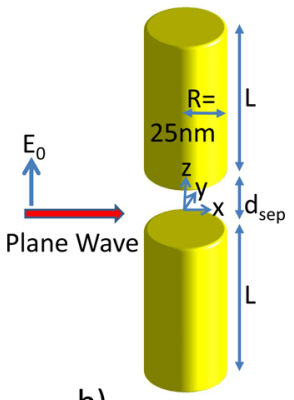

b)
Figure 1. Schematic of the gap antennas for (a) the spherical-gap and (b) the flat-gap configurations. The rods forming the antennas are rotationally symmetric with respect to the $z$ axis. The length of the rods is $L$ from end to end, and, except at the terminations, each rod has a radius $R$. The radius of the spherical cap near the gap in (a) is also $R$. The flat ends of the rods at the gap in (b), and at the outer ends for both (a) and (b), have radius $R^{\prime}$ and are rounded at the edge using a small radius of curvature $r ; R^{\prime}=R-r$. The structures are illuminated laterally, with the electric field of amplitude $E_{0}$ polarized along the rod axes and the Poynting vector, indicated by a red arrow, toward positive $x$. The center of the gap is at $x=y=z=0$, and the geometry has mirror symmetry with respect to the $z=0$ plane. When metallic antennas are considered, $L=100 \mathrm{~nm}, R=25 \mathrm{~nm}$, and $r=2$ $\mathrm{nm}$. For phononic $\mathrm{SiC}$ antennas $L=1500 \mathrm{~nm}, R=375 \mathrm{~nm}$, and $r=30$ nm.

nanorods as the arms of the antenna, separated by a gap distance $d_{\text {sep }}$ measured between the closest points. Each rod is of length $L$ and radius $R$, with a flat termination at the outer end far from the gap. The shape of the rod terminations at the gap determines the two types of antennas that we consider. In spherical-gap antennas (Figure 1a), each rod is terminated at the gap by a hemispherical cap and the contact is a zerodimensional point. In flat-gap antennas (Figure $1 \mathrm{~b}$ ), the corresponding termination is flat, with a two-dimensional contact area. The structures are rotationally symmetric with respect to the $z$ axis and have mirror symmetry with respect to the $z=0$ plane. The center of the gap corresponds to $x=y=z$ $=0$. The radii of all flat ends are $R^{\prime}=R-r$, with $r$ being a small edge rounding that prevents unrealistically sharp edges. When the flat-gap antenna is in contact $\left(d_{\text {sep }}=0\right)$, a small indentation occurs at the center of the antenna, but this indentation has no significant effect on the optical response under study.

The structure is placed in a vacuum, with permittivity $\varepsilon_{0}$, and is illuminated with light of angular frequency $\omega$, or equivalently wavelength $\lambda$, by a plane wave propagating along $x$ and with electric field of amplitude $E_{0}$ polarized along the rod axis $z$. We focus on metallic rods with $L=100 \mathrm{~nm}, R=25 \mathrm{~nm}$, and $r=2$ $\mathrm{nm}$, except in the section where $\mathrm{SiC}$ phononic antennas are considered and in Figure 6, where we vary the length of the metallic rods. The metal permittivity $\varepsilon_{\mathrm{m}}$ is modeled using a local Drude model; $\varepsilon_{\mathrm{m}}=\varepsilon_{0}\left[\varepsilon_{\infty}-\omega_{\mathrm{p}}^{2} /(\omega(\omega+\mathrm{i} \gamma))\right]$ with no dielectric background screening, $\varepsilon_{\infty}=1$, and plasma frequency and damping constants $\omega_{\mathrm{p}}=7.9 \mathrm{eV}$ and $\gamma=0.09 \mathrm{eV}$, respectively. These parameters correspond to a jellium model of gold and describe reasonably well the Au dielectric values for large wavelengths. The simple Drude model description facilitates the interpretation of the modes at large energies, where d-electron contributions can weaken the plasmonic resonances for real metals.

\section{NANOMETRIC GAP-NANOANTENNAS: SPHERICAL VERSUS FLAT GAPS}

Far-Field and near-Field Optical Response. We first analyze the local classical optical response of gap-antennas for gap separations $d_{\text {sep }} \geq 0.5 \mathrm{~nm}$. Nonlocal effects are known to influence the optical response in nanometric gaps, ${ }^{33-38}$ but the main trends of the modal structure and spectral dispersion in nanometric gaps can be described by local theories with a convenient rescaling of distances of a few ångstroms. ${ }^{39-41}$ For the range of gap separations considered, quantum tunneling effects can be neglected. ${ }^{26,32}$ Quantum effects for narrower gaps will be analyzed latter on.

We calculate the far- and near-field response, i.e., the extinction cross section $\sigma_{\text {ext }}$ and the maximum field enhancement at the central plane $\left|E^{\mathrm{max}} / E_{0}\right|$ (defined as the maximum amplitude of the field at the central plane $z=0$ normalized by the amplitude of the incident plane wave), by rigorously solving Maxwell's equations using the boundary element method (BEM). ${ }^{42}$ To diminish edge effects for the flat terminations, we consider only field enhancements for $x^{2}+y^{2}<(R-2 r)^{2}$.

We show the plasmonic response of spherical-gap antennas for different gap separations $d_{\text {sep }}$ in Figure 2a,c. For large gap

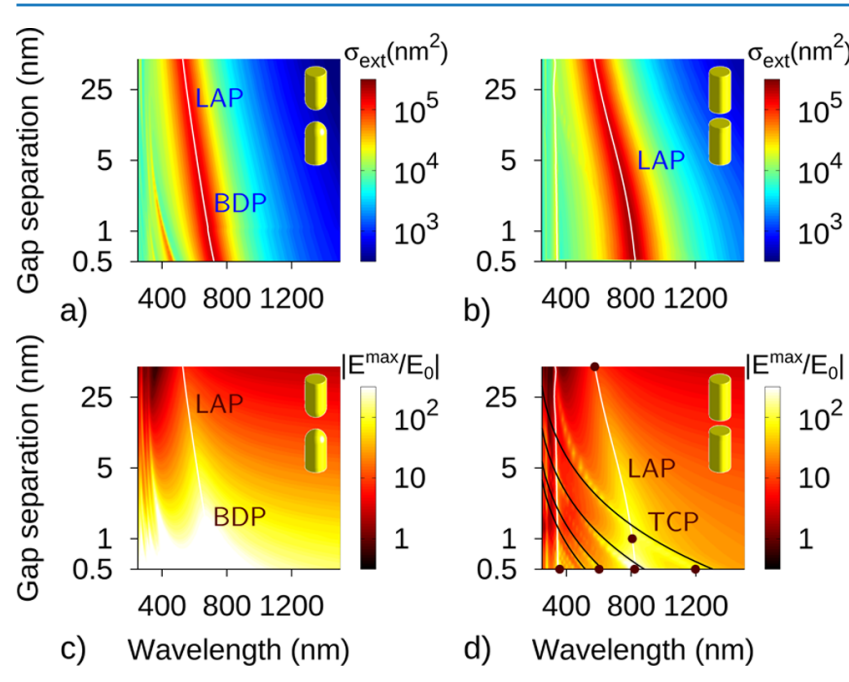

Figure 2. (a, b) Extinction cross-section $\sigma_{\text {ext }}$ and (c, d) maximum nearfield enhancement at the central plane $\left|E^{\max } / E_{0}\right|$ for $(\mathrm{a}, \mathrm{c})$ spherical-gap and (b, d) flat-gap linear metallic antennas, as a function of wavelength and gap separation. The white lines correspond to extinction maxima, and the black-lines in (d) to the expected position of the transverse gap cavity modes $(m=1, s=1)$ and $(m=0, s=2,4,6)$ according to the model given in the text. Notice the logarithmic scale used for the distances and for the color scale in these figures. The dots in (d) correspond to the field plots shown in Figure 5. Modes are labeled as longitudinal antenna plasmons (LAPs), transverse cavity plasmons (TCPs), and bonding dimer plasmons (BDPs).

separations, the dominant feature is a broad mode at approximately $\lambda=530 \mathrm{~nm}$ that can be associated with a localized surface plasmon in each antenna arm, oscillating along the antenna axis. We denote such excitations as longitudinal antenna plasmons (LAPs). As the gap separation decreases, the results follow the well-known trend for sphere dimers at small separations. ${ }^{8,10,17,43}$ The lowest energy LAP turns into a 
bonding dimer plasmon (BDP) mode, and higher order bonding resonances appear. All modes red-shift for decreasing separation distance, and the red-shift diverges (notice the logarithmic scale for separation distances) for $d_{\text {sep }} \rightarrow 0$, as a consequence of the hybridization of modes ${ }^{44}$ induced by Coulomb coupling of the charges in the individual arms.

All observed resonances are strongly radiative and lead to increasingly large near-field enhancement for smaller $d_{\text {sep }}$. As a consequence, both the extinction cross-section $\sigma_{\text {ext }}$ (Figure 2a) and the maximum local field enhancement $\left|E^{\max } / E_{0}\right|$ (Figure 2c) exhibit similar modal structure and dispersion. For very short $d_{\text {sep }}$, the field enhancement, produced by a large charge density at the apex of the rods, is confined to a very small volume within the center of the gap. $17,27,45$

The evolution of the optical spectra of flat-gap antennas as a function of separation, shown in Figure $2 \mathrm{~b}, \mathrm{~d}$, is strikingly different. The main feature in the extinction cross-section of Figure $2 \mathrm{~b}$ is a broad peak appearing in the wavelength range $\lambda$ $\approx 575 \mathrm{~nm}$ to $830 \mathrm{~nm}$. Additional narrow peaks at $\lambda \approx 340 \mathrm{~nm}$ and $\lambda \approx 280 \mathrm{~nm}$ are also found. These three modes can be understood as LAP resonances that behave as coupled FabryPérot-like resonances of the individual rods for plasmons propagating along the longitudinal $z$ direction. ${ }^{46-49}$ The coupling does not significantly affect the spectral positions of the two narrow LAP peaks at higher energy, but leads to a significant red-shift with decreasing $d_{\text {sep }}$ of the dominant, lowest energy LAP. Critically, and in contrast with the spherical-gap terminations, the red-shift saturates as the gap narrows ${ }^{24,50}$ toward the contact $d_{\text {sep }}=0$ limit, where the lowest energy LAP mode for a single nanorod of length $2 L$ is recovered.

We can observe more clearly the distinctive dispersion of the lowest energy LAP as $d_{\text {sep }} \rightarrow 0$ in Figure 3 where the influence

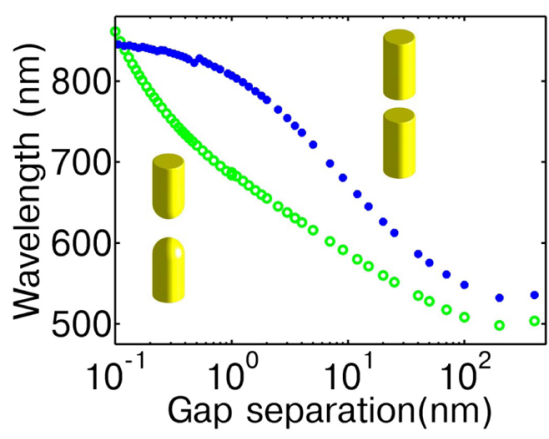

Figure 3. Resonant wavelength as a function of gap separation $d_{\text {sep }}$ for the lowest energy antenna mode (LAP) for both spherical (green) and flat gaps (blue), corresponding to the rightmost white lines in Figure 2.

of the gap morphology is emphasized. The diverging red-shift for narrow spherical gaps strongly contrasts with the saturation observed for the flat-gap antennas. To better show the trends, we include in Figure 3 also classical results for very narrow gaps up to $d_{\text {sep }} \approx 0.1 \mathrm{~nm}$, where electron tunneling can strongly modify the plasmonic response (see below). We can understand the saturation of the LAP by a simplified model that reduces the antenna to a simple electric circuit in series, ${ }^{51-53}$ where the gaps are represented by a capacitance and the rods by impedances. As the gap closes, the capacitance becomes divergently large and the gap can be seen as a short-circuit that does not affect the response, recovering the resonance of a single rod of twice the length. A more detailed and quantitative analysis is included as Supporting Information.
Remarkably, an additional set of modes is evident in the near-field response of the flat-gap antennas in Figure 2d. These modes (black lines) are spectrally narrow and exhibit a very strong dependence on gap separation. They can be clearly distinguished as strong enhancements for narrow nanometric gaps and radiate only weakly, ${ }^{54,55}$ thus hardly affecting the extinction cross-section in Figure $2 \mathrm{~b}$. We denote these resonances as transverse cavity plasmons (TCPs) because they can be understood as Fabry-Pérot-like resonances ${ }^{47,56}$ of cavity plasmons propagating along the gap cavity, similar to those found in patch or related plasmonic resonators. ${ }^{2,57-60}$ The surface plasmons excited at the metal-insulator-metal gap propagate parallel to the flat interfaces and reflect at the gap edges, ${ }^{61}$ leading, due to coherent interference, to resonances with characteristic standing-wave patterns.

An interesting property of the TCPs in our structures is that they interact only weakly with the lowest-energy LAP mode: indeed any possible anticrossing of TCPs and this LAP mode is lifted (and therefore observed as a crossing) because the losses of the modes are larger than the interaction strength. This property suggests that the LAP and the TCPs can be tuned independently by changing the geometry of the antenna arms or by altering the gap morphology. The excitation of the two states during the crossing of the TCP and LAP modes results in strong near-field enhancement when both overlap spectrally. It thus appears possible to tune the resonant energy of a TCP by a convenient cavity design and, at the same time, to control the strength of this mode by tuning the low-energy LAP mode via the geometry of the rest of the antenna.

We notice that the saturation of the lowest energy longitudinal mode $\mathrm{LAP}_{1}$ for the flat-gap antenna appears to correlate with the crossing with the transverse cavity mode $\mathrm{TCP}_{1}$. It would thus be tempting to ascribe this saturation to an anticrossing behavior. However, we have extensively investigated the charge distributions around the crossing point in a quasistatic approximation and find no evidence of the change of mode symmetry one would expect from an anticrossing.

Longitudinal Antenna Plasmons and Transverse Cavity Plasmons. We now discuss in more detail the nature of the modes of the flat-gap antennas. For the present geometry, all plasmon modes can be classified according to their azimuthal symmetry $m$ describing the variation of the charge densities under rotation around the $z$ axis. In the present situation, only $m=0$ and $m=1$ modes play a role. The longitudinal antenna modes $(m=0)$ of the single isolated rods can be classified by $l \geq 1$, where $l$ is the number of nodes in the charge distribution along the antenna axis (longitudinal direction). ${ }^{15}$ Smaller $l$ corresponds to lower energy (longer wavelength) modes. The associated charge density patterns resemble those of traditional linear antennas operating at much lower frequencies. ${ }^{62}$ Similarly, the TCPs can be labeled by $s \geq 1$ according to the number of charge nodes along the transverse $x$ axis parallel to the propagation direction of the incident light. Odd $s$ corresponds to $m=1$ and even $s$ to $m=0$.

We summarize the evolution of the flat-gap LAPs and TCPs as the gap separation is decreased in Figure $4 \mathrm{a}$ and illustrate their corresponding symmetries in terms of $l$ and $s(m)$ numbers in the schematics of Figure $4 \mathrm{~b}$. The low-order LAP $(m=0, s=0)$ mode goes through a transition from the $l=1$ modes of the individual rods when the gap separation is large, to a $l=1$ mode of the $2 L$ full antenna after contact (see top schematics of the charge densities in Figure $4 \mathrm{~b}$ ). This transition 


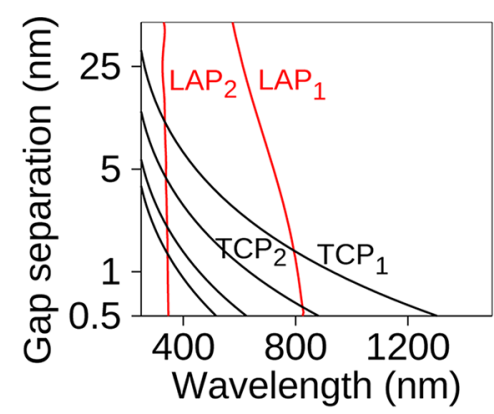

a)

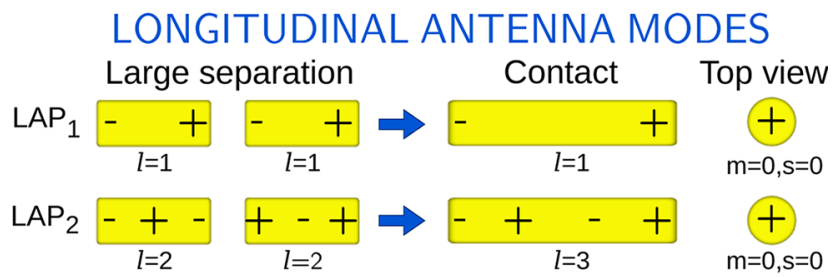

TRANSVERSE CAVITY MODES Large separation
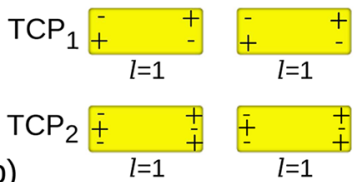
Short distance

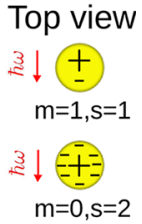

Figure 4. Schematic of the evolution of the modes in flat-gap antennas. The top panel (a) shows the spectral evolution (extinction maxima) of the LAPs (red) and the position of the $s=1,2,4$, and 6 TCPs predicted by the model in the text (black), consistent with the modes obtained in Figure 2d. The charge distributions for the two lowest energy LAPs (labeled $\left.\mathrm{LAP}_{1}, \mathrm{LAP}_{2}\right)$ and TCPs $\left(\mathrm{TCP}_{1}, \mathrm{TCP}_{2}\right)$ are sketched in (b), where we also classify them according to the nomenclature $(l ; \mathrm{s}, m)$ introduced in the text. The signs at the ends of the rods refer to charges at the flat vacuum-metal interfaces, and those along the rods to charges at the lateral side-walls. The symmetry of the mode on the horizontal plane $(s, m)$ does not depend on the gap distance. All modes shown are rotationally symmetric except for the TCP $(m=1, s=1)$, for which we indicate explicitly how the charge distribution relates to the direction of the incident light. results in a significant red-shift of the plasmonic resonance, up to the saturation occurring for very narrow gaps (Figure 3 ).

Similarly, the $l=2$ mode of the single rods becomes the $l=3$ LAP mode of the full antenna $\left(\mathrm{LAP}_{2}\right.$, bottom schematics of the longitudinal antenna modes in Figure $4 \mathrm{~b})$. As a general rule, the $l$ modes of the short antennas become the $(2 l-1)$ LAP mode of the large joined antenna. The spectral position of the $l=2,3$ modes at large distance and the corresponding $(2 l-1)$ resonance of the full structure after contact is very similar for our system, so that the resulting spectral shift is small (resonances at $\lambda \approx 340 \mathrm{~nm}$ and $\lambda \approx 280 \mathrm{~nm}$ ). The $l=2$ of the individual rods at $\lambda \approx 340 \mathrm{~nm}$ are only excited due to their mutual interaction, as they would otherwise be symmetryforbidden under the current illumination conditions.

To corroborate our understanding of the symmetry of the longitudinal modes, we plot the near-field distribution of the lowest energy LAP in Figure 5a,b for two separation distances. The $l=1$ field distribution shows the characteristics of a bonding combination of individual rod dipolar modes interacting at large separation distances (Figure 5a) and a $l=$ 1 mode of the combined structure for narrow gaps (Figure $5 b$ ).

The TCP modes (black lines in Figure 4a) are characterized by a strong dispersion with the gap separation. Many TCPs are apparent in the near-field spectrum for very narrow (strongly interacting) gaps. Each mode corresponds to different orders of plasmonic Fabry-Pérot-like cavity modes at the gap, as depicted in the bottom schematics of Figure $4 \mathrm{~b}$, where the charge density patterns of the $(m=1, s=1)$ and $(m=0, s=2)$ modes are shown. These modes are strongly coupled within the gap and are very weakly radiative. To illustrate the nature of the TCPs, we plot in Figure $5 \mathrm{c}-\mathrm{f}$ the spatial distribution of the electric field amplitude in the middle gap plane $(z=0)$ for a gap separation $d_{\text {sep }}=0.5 \mathrm{~nm}$ and several orders of $s$. The field distributions in Figure $5 \mathrm{c}-\mathrm{e}$ correspond to $s=1,2,4$, respectively. As expected, clear standing-wave patterns typical of transverse Fabry-Pérot resonances can be observed. Higher order TCPs reveal a progressively larger number of maxima and minima that corresponds to an increasing cavity order $s$. For example, Figure $5 \mathrm{f}$ corresponds to a large $s$ value of $\sim 14$, illustrating the very fast spatial field variation of these highenergy modes.
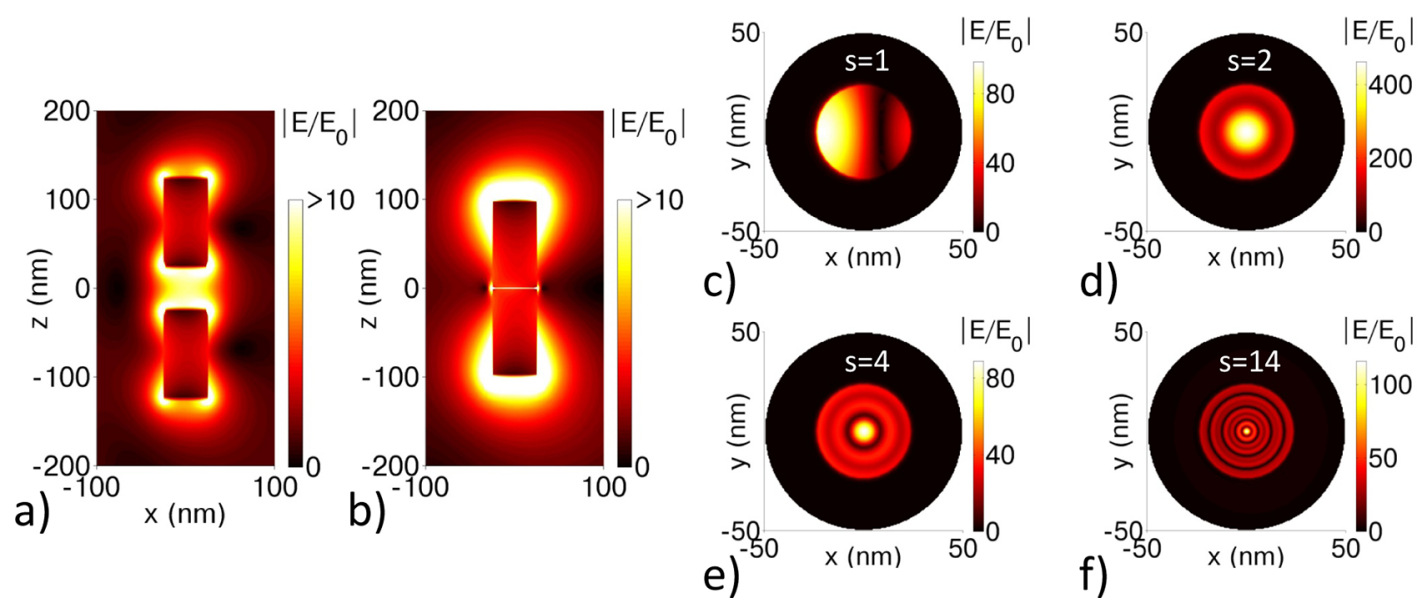

Figure 5. Normalized electric field $\mid E / E_{0} l$ in the $(\mathrm{a}, \mathrm{b})$ vertical plane $(y=0)$ and $(\mathrm{c}-\mathrm{f})$ horizontal $x y$ plane at the gap center $(z=0)$ for the flat-gap antenna. (a) $d_{\text {sep }}=50 \mathrm{~nm}, \lambda=575.5 \mathrm{~nm}$, (b) $d_{\text {sep }}=1 \mathrm{~nm}, \lambda=807 \mathrm{~nm}$, (c) $d_{\text {sep }}=0.5 \mathrm{~nm}, \lambda=1197 \mathrm{~nm},(\mathrm{~d}) d_{\text {sep }}=0.5 \mathrm{~nm}, \lambda=821.5 \mathrm{~nm},(\mathrm{e}) d_{\text {sep }}=0.5$ $\mathrm{nm}, \lambda=602.0 \mathrm{~nm}$, (f) $d_{\text {sep }}=0.5 \mathrm{~nm}, \lambda=358.5 \mathrm{~nm}$. These conditions are also indicated by the dots in Figure $2 \mathrm{~d}$. (a) and (b) correspond to the lowest energy LAP and (c)-(f) to TCPs $(m=1, s=1)$ and $(m=0, s=2,4$, and $\sim 14) . x, y$, and $z$ refer to the coordinate axes in Figure 1 . 
Modes of even $s$ correspond to rotationally symmetric fields $(m=0)$, while modes with odd $s$ also present a variation in the $x y$ plane in the azimuthal direction $(m=1)$. The latter resonances are excited only due to retardation, which breaks the symmetry between positive and negative $x$. Only the $(m=1, s=$ 1 ) odd mode exhibits a significant field enhancement in our geometry. This $(m=1, s=1)$ resonance should ideally show a perfect antisymmetric field distribution with respect to the $x=$ 0 plane but appears asymmetric due to the contribution to the field from the relatively broad $\mathrm{LAP}_{1}$ mode and from nonresonant contributions. ${ }^{63}$ Flat-gap antennas can also support higher $m$ modes corresponding to faster variations on the azimuthal direction, ${ }^{58}$ but they do not seem to contribute to the response in our scenario.

Traditionally, transverse modes in nanorods refer to modes that are excited by a field polarized transversally to the nanorod axis. The TCPs discussed here are transverse in the sense that their surface charge distribution has significant variation with nodes and antinodes in the transverse direction along the gap faces. The even $s$ TCPs cannot, by symmetry, be excited by a transversely polarized field. They are only excited by a longitudinal field. In that sense, they must evolve as the gap closes from high-order longitudinal modes of the separated nanorods. These TCPs become visible, among other factors, because of the strong interparticle coupling for small gaps. The coupling between the $m=0$ TCP modes and the LAP modes likely depends on the gap separation and the size of the individual nanorods. The $(m=1, s=1)$ TCP is constructed from modes of the separated nanorods that are dark for both transverse and longitudinal polarization, as observed from our quasistatic calculations (not shown). Retardation effects turn this TCP into a bright mode for longitudinal polarization but remains dark for transverse polarization.

The relevant TCPs for different values of $s(m=0$ and $m=$ 1) can be described by a simple Fabry-Pérot-like model. We first obtain the plasmonic wavelength $\lambda_{\mathrm{pl}}$ of the cavity gap mode propagating along a thin infinite vacuum gap layer between two semi-infinite metallic slabs. ${ }^{56,64,65}$ When the equations of propagation in this metal/insulator/metal system are solved for a given incident wavelength $\lambda$ and gap separation $d_{\text {sep}}$, one obtains: ${ }^{66}$

$$
\tanh \left(\mathrm{i} \pi d_{\mathrm{sep}} \sqrt{\frac{1}{\lambda^{2}}-\frac{1}{\lambda_{\mathrm{pl}}^{2}}}\right)=\frac{\varepsilon_{0}}{\varepsilon_{\mathrm{m}}} \sqrt{\frac{\varepsilon_{0} \lambda^{2}-\varepsilon_{\mathrm{m}} \lambda_{\mathrm{pl}}^{2}}{\varepsilon_{0} \lambda^{2}-\varepsilon_{0} \lambda_{\mathrm{pl}}^{2}}}
$$

which could straightforwardly be generalized to account for a dielectric material in the gap by substituting the wavelength $\lambda$ and the permittivity in a vacuum with the respective values in the dielectric. A TCP appears when the incident illumination excites a plasmon with wavelength $\lambda_{\mathrm{pl}}$ that approximately satisfies the quantization condition $R^{\prime} / \lambda_{\mathrm{pl}}=s / 4$, which connects $\lambda_{\mathrm{pl}}$ with the cavity radius $R^{\prime}$ and the order $s \geq 1$ of the cavity mode. This quantization condition can be obtained by considering a single gap plasmon that propagates in the transverse $x$ direction between the edges of the flat gap along a diameter of length $2 R^{\prime}$. A resonance occurs when the phase accumulated by the plasmon after a full round trip is $2 \pi$ times the integer $s$ order; $\left(2 \pi / \lambda_{\text {pl }}\right) 4 R^{\prime}=2 \pi s$. Similar equations were found for other plasmonic cavities, although an additional phase term was often considered $47,54,56,67,68$ and more complex equations have also been studied. ${ }^{60,69,70}$ The results of the spectral position of the TCPs given by this simple analytical model for $s=1,2,4$, and 6 are superimposed to the calculated field enhancement in Figure $2 \mathrm{~d}$ as black solid lines. The agreement between the positions of the TCPs as predicted from the model and the calculations is remarkable and indeed suggests a spectral decoupling of the two types of resonances.

Our simple model also explains the large sensitivity of the TCP modes' resonant frequency to the gap separation. In the limit of no losses, $\lambda_{\mathrm{pl}} \ll \lambda$ and $\varepsilon_{\mathrm{m}} / \varepsilon_{0} \approx-\lambda^{2} / \lambda_{\mathrm{p}}^{2}$; then eq 1 can be reduced to $\lambda_{\mathrm{pl}}\left(1+\left(\lambda_{\mathrm{pl}} / \lambda_{\mathrm{p}}\right)^{2}\right)^{1 / 2} \approx \pi d_{\text {sep }} \lambda^{2} / \lambda_{\mathrm{p}}^{2}$, where $\lambda_{\mathrm{p}}$ is the plasma wavelength associated with $\omega_{\mathrm{p}}, \lambda_{\mathrm{p}}=2 \pi c_{0} / \omega_{\mathrm{p}}$, and $c_{0}$ is the speed of light in a vacuum. The resonant wavelength $\lambda_{\text {res }}$ is then found near $\lambda_{\text {res }} \approx \lambda_{\mathrm{p}}\left(4 R^{\prime} /\left(\pi d_{\text {sep }} s\right)\right)^{1 / 2}\left[1+\left(4 R^{\prime} /\left(s \lambda_{\mathrm{p}}\right)\right)^{2}\right]^{0.25}$. This expression shows that the resonant wavelength scales with $\lambda_{\text {res }} \propto 1 / \sqrt{ } d_{\text {sep }}$ as $d_{\text {sep }} \rightarrow 0$.

Although the length $L$ of the rods critically affects the LAPs, they do not appear in this simple model for the TCPs. Figure 6
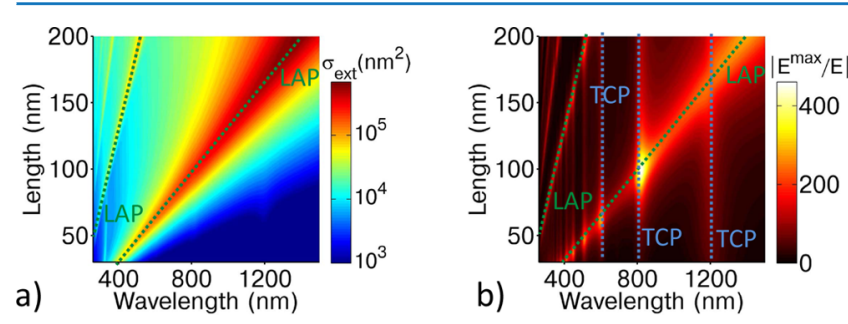

Figure 6. (a) Extinction cross-section $\sigma_{\text {ext }}$ and (b) maximum near-field enhancement at the central plane $\left|E^{\max } / E_{0}\right|$ for flat-gap metallic antennas, as a function of wavelength and length of the rods $L$. The gap separation is $d_{\text {sep }}=0.5 \mathrm{~nm}$. The dotted lines are guides to the eye indicating the evolution of some of the LAPs (oblique green lines) and TCPs (vertical blue lines).

emphasizes the very different effect of $L$ on the two sets of modes, for a narrow $d_{\text {sep }}=0.5 \mathrm{~nm}$ gap. The far field in Figure 6a shows LAPs that red-shift clearly as the length increases (green lines) and that are also visible in the near field (Figure 6b). The TCPs are identified by the narrow lines in the near-field enhancement (Figure 6b), whose spectral position is, as expected, essentially independent of the rod lengths (vertical blue lines). In general, particularly large enhancements are obtained for a given TCP (most notably for even TCPs), when the LAP mode overlaps the TCP.

Figure 6 thus illustrates that it is possible to control the strength of the TCPs, without affecting their spectral position, by tuning the longitudinal antenna resonances through $L$. Complementarily, Figure 2 showed how the gap separation distance controls the resonant position of the TCPs but does not influence the LAPs for narrow gaps.

\section{SUBNANOMETER GAPS: THE TUNNELING REGIME}

The results discussed up to now employed classical modeling of the antenna response. However, when the distance of the gap between the nanoantennas reaches subnanometric dimensions, quantum effects ${ }^{71-73}$ such as tunneling of electrons through the gap start to play an important role for the optical response of the system. ${ }^{26-28,74}$ To model this effect, we use the recently developed quantum corrected model ${ }^{32}$ within a local framework. The QCM amounts to the insertion of an effective material in the gap, with a conductance obtained from a quantum mechanical calculation of the tunneling probability across a metallic gap. ${ }^{32,41}$ The tunneling and thus the conductivity of the associated effective material depend on the distance between the opposite surfaces bridging the gap and will vary laterally across a gap of arbitrary shape, depending on 
the local separation distance at each point of the gap. The QCM approach thus makes it possible to model the system using a classical electrodynamical solver and account for quantum mechanical electron tunneling effects.

In Figure 7 we plot the extinction cross-section for sphericalgap antennas (Figure 7a,c) and flat-gap antennas (Figure $7 \mathrm{~b}, \mathrm{~d}$ )
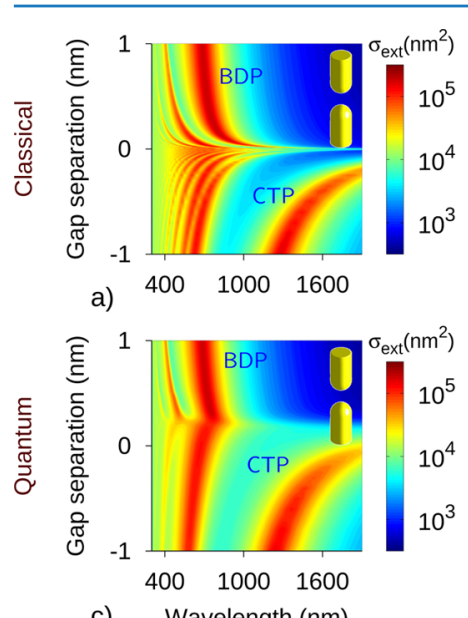

)
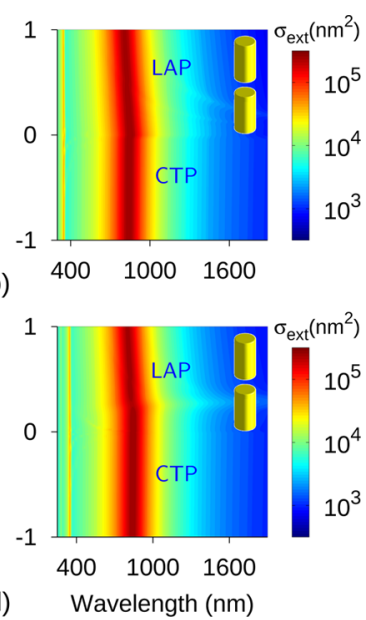

Figure 7. Extinction cross-section $\sigma_{\text {ext }}$ of metallic gap-antennas as a function of the wavelength and gap separation $d_{\text {sep }}$ for (a, c) spherical and $(b, d)$ flat terminations obtained using $(a, b)$ classical calculations and (c, d) the QCM. Some longitudinal antenna plasmons (LAPs), bonding dimer plasmons (BDPs), and charge transfer plasmons (CTPs) are labeled in the plots. Linear separation distance scale is used in all of these figures. Notice the logarithmic scale used for the color coding in all the figures. Negative distances correspond to overlapping structures.

as a function of the gap separation and wavelength using both classical (Figure 7a,b) and QCM (Figure 7c,d) calculations. As tunneling only becomes significant for distances $\lesssim 0.5 \mathrm{~nm}$, ${ }^{32}$ we focus here on very narrow gaps of $d_{\text {sep }}<1 \mathrm{~nm}$. We also consider negative separation distances, corresponding to the case of overlapping antenna arms. A negative distance $d_{\text {sep }}$ corresponds to separation $L-\left|d_{\text {sep }}\right|$ between the center of the two rods.

The classical results for the spherical case (Figure 7a) before contact reproduces the behavior already observed in Figure 2a for larger gap separations, emphasizing the divergent red-shift of the different bonding modes as the gap closes. An unphysical discontinuity is present at contact $\left(d_{\text {sep }}=0\right)$, separating the nontouching and the overlapping regimes. ${ }^{17}$ For $d_{\text {sep }}<0$ several charge transfer plasmons ${ }^{18,75}$ (CTPs) appear in the spectrum, blue-shifting with increasing overlap. The CTPs are plasmonic modes that emerge when charges can be transferred from one antenna arm to the other. The lowest energy CTP is excited at a characteristic low energy.

The QCM results for the spherical-termination antennas (Figure 7c) display trends consistent with previous work. ${ }^{26,28,76}$ Instead of a discontinuity at contact we find a gradual transition between the nontouching and the overlapping regimes with a distinctive change from modes that red-shift as the gap narrows to modes that blue-shift with further decrease of $d_{\text {sep. The }}$ change occurs near a threshold distance $d_{\text {th }} \approx 0.2$ to $0.3 \mathrm{~nm}$ that can be understood as the separation where the amount of charge being transported across the gap in a fraction of the optical period is comparable to the charges induced at the metal interfaces at the gap. ${ }^{18,27}$ For $d_{\text {sep }} \gtrsim d_{\text {th }}$ the modes are fundamentally the same as those obtained from the classical calculations. For $d_{\text {sep }} \lesssim d_{\text {th }}$, charge transfer is allowed and the modes can be considered as CTPs even if physical contact has not been established. For overlapping arms $\left(d_{\text {sep }}<0\right)$, we obtain significantly fewer CTPs in the presence of tunneling compared to the classical results, which can be related to a softening of the contact regions between the two rods due to charge transfer in the quantum treatment.

The far-field spectra for narrow flat gaps in Figure $7 b, d$ behave very differently. The extinction cross-section calculated using the classical and QCM approach is very similar and insensitive to the gap separation. In both cases, the gap behaves as a short circuit, and the response corresponds to that of a single rod of approximate length $2 L$. In the classical case, this short circuit is due to physical contact $\left(d_{\text {sep }}<0\right)$ or to a large capacitance $\left(d_{\text {sep }}>0\right.$, see Supporting Information). In the QCM electron tunneling just assists in reducing the gap impedance for $d_{\text {sep }}>0$. To consider the gap morphology is thus relevant to properly associate quantum effects such as tunneling with a particular charge transfer modal structure. ${ }^{29}$

Figure 8 shows the maximum field enhancement $\left|E^{\max } / E_{0}\right|$ for both types of antennas as a function of the gap separation and

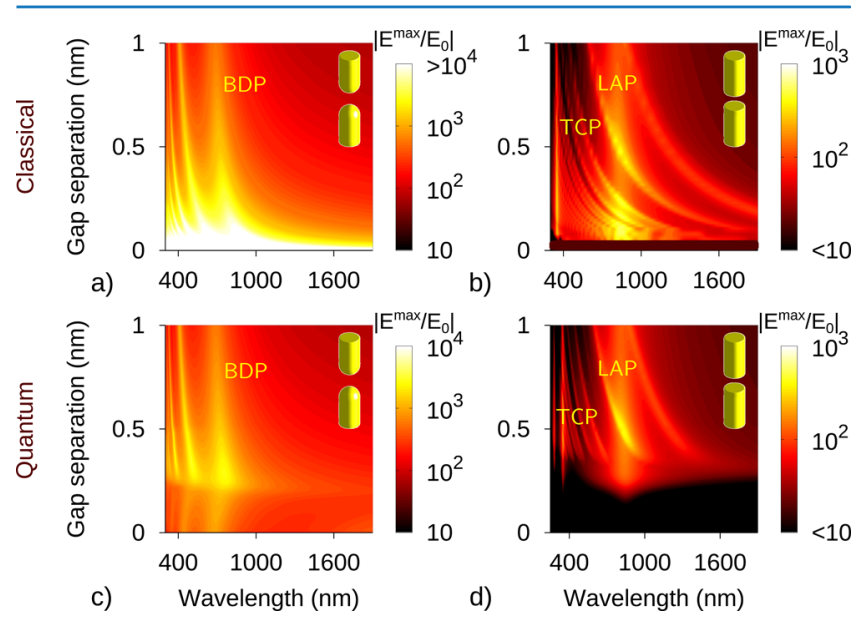

Figure 8. Maximum electric field enhancement $\left|E^{\max } / E_{0}\right|$ at the central plane of a metallic antenna gap as a function of the illumination wavelength and the separation distance, $d_{\text {sep }}$, for (a, c) spherical and (b, d) flat gaps obtained using (a, b) classical calculations and (c, d) the QCM. Some of the longitudinal antenna plasmons (LAPs), bonding dimer plasmons (BDPs), and transverse cavity plasmons (TCPs) are labeled in the plots. Linear separation distance scale is used in all of these figures. Notice the logarithmic scale used for the color coding in all the figures.

wavelength, for positive separation distances $d_{\text {sep }}>0$. The classical results (Figure 8a,b) show the same tendencies as those in Figure $2 \mathrm{c}, \mathrm{d}$ for larger separation. In particular the coexistence of TCPs and LAPs for flat gaps is apparent. The field enhancement produced at the spherical gap is noticeably larger than for the flat counterpart due to a more singular concentration of the field in that geometry.

The QCM results in Figure 8c,d show that the electric fields in the gap become quenched for gap separations below a few angstroms because of the onset of electron tunneling. ${ }^{26,76}$ The charge transfer across the gap counteracts the strong charge pile-up predicted by the classical description and short-circuits the gap. The electric field quenching for flat-gap termination (Figure $8 \mathrm{~d}$ ) occurs at longer separation distances than for the spherical case. For flat gaps the quenching of the TCPs occurs 
for gap separations of $d_{\text {sep }} \approx 0.4 \mathrm{~nm}$, while that for spherical terminations at $d_{\text {sep }} \approx 0.3 \mathrm{~nm}$, predicting a large sensitivity of the TCPs to tunneling. Thus, our results for flat narrow gaps indicate that, although in this case the tunneling does not affect the extinction cross-section, which is dominated by the global current flow in the vertical direction, a quantum mechanical description is essential for the modeling of the local charge concentrations and thus the local field enhancements.

\section{PHONONIC GAP-ANTENNAS: MORPHOLOGY EFFECTS IN THE INFRARED}

In flat metallic antennas a large number of cavity TCP modes were predicted, but most of them emerged clearly only for very narrow gaps of around $1 \mathrm{~nm}$ or less. Such narrow gaps were also necessary to obtain the characteristic saturation behavior with vanishing separation distance of the lowest energy antenna LAPs. Despite significant recent experimental progress, ${ }^{15,21,27-29,77-82}$ fabrication of such narrow gaps remains an experimental challenge. Furthermore, in realistic metals, the influence of d-electrons can introduce significant losses for frequencies near the surface plasmon resonance, with a detrimental effect for the excitation of well-defined high-order TCPs. Two possibilities to better access the TCPs are to use wider metallic structures or to fill the gap with a dielectric material that establishes a physical spacer. ${ }^{83-85}$

These challenges can be mitigated when the size of the structures is scaled up to micrometer-sized dimensions. We now analyze gap-antennas made of polar materials, such as $\mathrm{SiC}$, which support localized surface phonon-polariton resonances that can be efficiently coupled to infrared (IR) radiation. The behavior of phononic antennas in the infrared is analogous to the plasmonic response of metallic antennas in the visible, since the optical response of polar materials in a range of infrared wavelengths is qualitatively similar to that of metals below the plasma frequency. ${ }^{25,86-88}$

The optical permittivity of $\mathrm{SiC} \varepsilon_{\mathrm{SiC}}$ can be modeled using a Lorentz-Drude response, $\varepsilon_{\mathrm{SiC}}=\varepsilon_{0} \varepsilon_{\infty}\left[1+\left(\omega_{1}^{2}-\omega_{\mathrm{t}}^{2}\right) /\left(\omega_{\mathrm{t}}^{2}-\right.\right.$ $\left.\left.\omega^{2}-\mathrm{i} \omega \Gamma\right)\right]$, where $\omega_{1}$ and $\omega_{\mathrm{t}}$ are the longitudinal and transverse phononic angular frequencies, respectively. ${ }^{89,90} \varepsilon_{\infty}$ sets the values at very large energies, and $\Gamma$ describes the losses in the material. For $\mathrm{SiC}$ we use ${ }^{89,91,92} \omega_{1}=969 \mathrm{~cm}^{-1}, \omega_{\mathrm{t}}=793$ $\mathrm{cm}^{-1}, \varepsilon_{\infty}=6.7$, and $\Gamma=4.76 \mathrm{~cm}^{-1}$. The frequencies where phononic antenna modes can occur are limited to the window between $\omega_{1}$ and $\omega_{\mathrm{t}}$, where the real part of $\varepsilon_{\mathrm{SiC}}$ is negative.

Figure 9 shows full electromagnetic calculations of the extinction (Figure 9a,b) and maximum near-field enhancement $\left|E^{\max } / E_{0}\right|$ at the central plane of the gap (Figure $9 \mathrm{c}, \mathrm{d}$ ) for $\mathrm{SiC}$ antennas with spherical (Figure 9a,c) and flat (Figure 9b,d) terminated gaps. As $d_{\text {sep }}$ is always relatively large, we can disregard quantum effects. ${ }^{93}$ The morphology considered is the same as for metallic antennas (Figure 1) after scaling up all the dimensions of the rods by a factor of $15(L=1500 \mathrm{~nm}, R=375$ $\mathrm{nm}$, and $r=30 \mathrm{~nm}$ ). The $\mathrm{SiC}$ antenna is resonant at wavelength $\approx 11.5 \mu \mathrm{m}$, and thus the dimensions normalized by the wavelength of the incident radiation are similar to those considered for our metallic antennas. In general, we find very similar trends to those for the metallic antennas. Since modes for $\mathrm{SiC}$ are phononic rather than plasmonic, we will label them using the previous scheme of notation but referring to the different types of modes as phononic $(\mathrm{Ph})$ instead of plasmonic (P).

For spherical gaps, we observe, both in the far (Figure 9a) and in the near field (Figure 9c), a set of phononic modes that
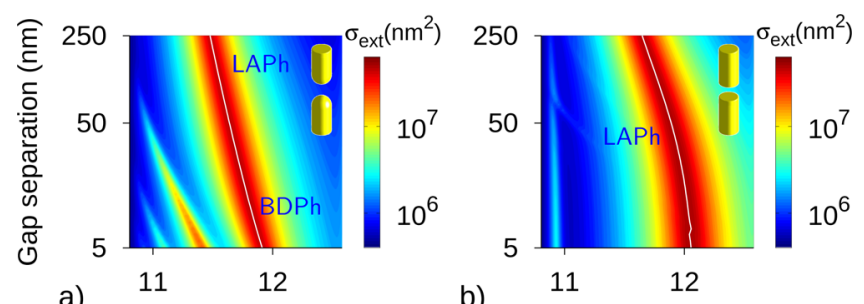

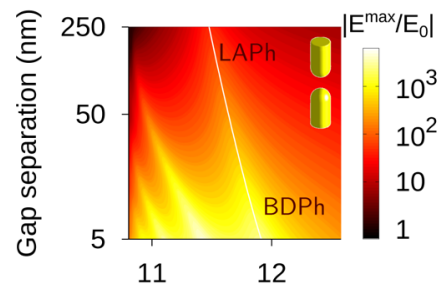

c) Wavelength $(\mu \mathrm{m})$ b)

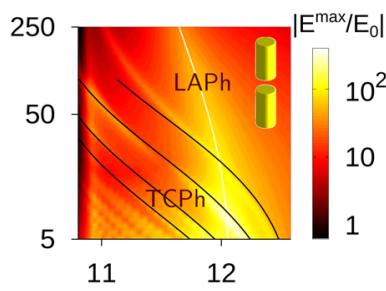

d) Wavelength $(\mu \mathrm{m})$
Figure 9. (a, b) Extinction cross-section $\sigma_{\text {ext }}$ and (c, d) maximum nearfield enhancement $\left|E^{\max } / E_{0}\right|$ at the central plane of the gap for (a, c) spherical-gap and (b, d) flat-gap antennas made with $\mathrm{SiC}$, as a function of wavelength and gap distance. The black lines in (d) correspond to the expected position of the phononic analogue to the TCPs, here denoted as TCPhs, with $s=1,2,4,6$ according to eq 1 . Notice the logarithmic scale used for the distances and for the color scale in all figures. Some longitudinal antenna phononic modes (LAPh), bonding dimer phononic modes $(\mathrm{BDPh})$, and transverse cavity phononic modes $(\mathrm{TCPh})$ are indicated in the panels.

red-shift considerably as the gap separation decreases. The near-field enhancement for short distances is very large. Since the phononic modes are confined to a relatively narrow spectral window $(10.3 \mu \mathrm{m} \lesssim \lambda<\lesssim 12.6 \mu \mathrm{m})$ where the real part of $\varepsilon_{\mathrm{SiC}}$ is negative, the red-shift of the bonding modes does not diverge for decreasing $\mathrm{SiC}$ gaps.

In contrast, for flat gaps, the red-shift of the broad longitudinal antenna phononic $\mathrm{LAPh}_{1}$ mode that dominates the extinction (Figure 9b) shows a clear saturation already for $d_{\text {sep }} \approx 10 \mathrm{~nm}$ and converges toward the $l=1$ resonance of the joint structure. The wavelength of the second-order extinction peak at $\lambda \approx 10.9 \mu \mathrm{m}$ is also weakly sensitive to $d_{\text {sep }}$ and corresponds to the $\mathrm{LAPh}_{2}$ mode evolving from the $l=2$ mode of the individual rods. The LAPhs show also a clear signature in the near-field spectrum, displayed in Figure 9d, where the TCPhs emerge as an additional set of narrow peaks that disperse strongly with separation distance and are very weakly radiative (almost not distinguishable in the extinction spectrum). In contrast to what was observed for the metallic antennas, in the $\mathrm{SiC}$ antenna many TCPhs are clearly visible for $d_{\text {sep }}$ around $10 \mathrm{~nm}$. We note that as for the metallic antennas, the saturation of the $\mathrm{LAPh}_{1}$ mode seems to coincide with its crossing of the $\mathrm{TCPh}_{1}$ mode, but, as pointed out in the plasmonic case, an analysis of the charge distributions of the modes in the quasistatic approximation reveals no signs of an anticrossing.

As the wavelength of the phononic modes in $\mathrm{SiC}$ is always smaller than $12.6 \mu \mathrm{m}$ (corresponding to $\omega_{\mathrm{t}}$ ), at a distance of about $d_{\text {sep }} \approx 10 \mathrm{~nm}$ the strong red-shift with decreasing gap of the lowest energy TCPs becomes less marked in comparison to the diverging red-shift observed in Figure 2 for metallic antennas. Nevertheless, as the distance decreases from hundreds to tens of nanometers, the red-shift of the TCPhs is very significant and also follows the Fabry-Pérot-like relationship $R^{\prime} / \lambda_{\text {ph }}=s / 4$ with $\lambda_{\text {ph }}$ given by eq 1 , as indicated 
by the black lines in Figure 9d. The phononic wavelength $\lambda_{\text {ph }}$ should be used instead of the plasmonic $\lambda_{\mathrm{pl}}$ value and $\varepsilon_{\mathrm{SiC}}$ instead of the $\varepsilon_{\mathrm{m}}$, but otherwise the equation describing the dispersion of the TCPhs remains the same. These results give evidence that polar gap-antennas such as those made of $\mathrm{SiC}$ could serve as candidates to control and tune separately cavity and antenna modes to obtain large field enhancements.

\section{SUMMARY AND DISCUSSION}

We have studied in detail the behavior of metallic antennas with very narrow flat gaps, contrasting it with the response for spherical terminations at the gap. The behavior predicted for the latter is similar to that for spherical particles, with a set of bonding modes that strongly red-shift with narrowing gaps and that determine both the near- and the far-field response. ${ }^{17}$ In contrast, for the corresponding flat-gap antennas, we found two different sets of modes, ${ }^{20,21,84}$ longitudinal antenna plasmons and transverse cavity plasmons, which behave in fundamentally different ways. The LAPs dominate the far-field response and saturate spectrally for narrow gaps. The saturation of the $\mathrm{LAP}_{1}$ can be qualitatively modeled using a circuit model where a capacitor represents the gap cavity. The TCPs are confined to the gap between the flat antenna arms and are thus very sensitive to separation distance $d_{\text {sep }}$ and can be described using a simple Fabry-Pérot-like model. The TCPs are essentially dark modes ${ }^{94}$ and have a negligible effect on the far-field optical properties. Both LAPs and TCPs contribute strongly to the near-field response, i.e., the plasmon-induced electric field enhancement. Most significantly, we find that the TCPs and the LAPs can be tuned independently. As a consequence, the spectral and spatial distribution of the near fields in the gap can be tuned by changing the structure of the gap. We also demonstrate the validity of these concepts for phononic SiC antennas in the infrared. The dependence on the gap morphology identified for metallic antennas is reproduced, but here occurs for significantly wider gaps around $10 \mathrm{~nm}$. The strong dependencies on the gap morphology are also manifested when electron tunneling is accounted for. Significant differences in the influence of charge transfer on the modal structure of charge transfer plasmons are found in this case, a situation that needs to be considered when interpreting molecular transport experiments dealing with the quantum regime. $^{29}$

The cavity modes can be supported by any plasmonic junction with flat faces, assuming that the lateral dimensions of the cavity are comparable to the plasmonic wavelength in the gap and that the separation distance is relatively small. For nearly flat gaps, even small variations of the gap separation across the faces may suppress the formation of these modes or confine them to particular regions of the gap. The responses for flat and spherical gaps are thus qualitatively different and cannot be explained simply by a stronger interaction in the flat configuration due to the larger amount of material near the gap.

Our results could contribute to an explanation of many discrepancies and inconsistencies in experiments that are sensitive to the fine details of the morphology of a plasmonic nanocavity. Morphological changes of the cavities at the nanometric level can significantly modify the performance of a particular optoelectronic process at the plasmonic interface, potentially explaining the often encountered variability in experimental results. Well-reproduced cavities are thus key to produce systematic experimental output. ${ }^{95}$ Notably, the exact field distribution in a plasmonic cavity determines the performance of many field-enhanced spectroscopies, such as in tip-enhanced Raman spectroscopy (TERS) ${ }^{96,97}$ and of scattering-type scanning near-field optical microscopy (sSNOM). ${ }^{98}$ The variation of the morphology of the tip-onsubstrate cavities in these spectroscopies can indeed explain the large tip to tip dependencies in signal quality and spectral behavior often found in experiments. ${ }^{99}$ Similarly, modifications of the gap can also determine the yields and properties of many optoelectronic processes such as in photoemission ${ }^{100,101}$ or in nonlinear plasmonics. ${ }^{102}$

However, the sensitivity to fine details of the cavity, even reaching down to atomic-scale variations within the gap, is not always detrimental. If controlled, as for example in a particleon-mirror geometry, the dependence on the faceting can serve as an optical monitor of complex photochemical processes ${ }^{85}$ or to trace transport properties at optical frequencies, ${ }^{29,103}$ thus allowing access to information that cannot be reached by other techniques.

Our results stress the importance of the morphology of the gap for applications in nanophotonics. The independent control of the two types of modes, as introduced here, provides a valuable means to engineer the far-field and near-field properties separately. Flat-gap termination antennas may find applications beyond the manipulation of light under plane-wave illumination. The spatial field distribution observed for the different cavity modes, often with closely spaced maxima and minima, may be interesting for controlling the coherent interaction between different emitters in a gap, with potential application in energy transfer ${ }^{104,105}$ or quantum information involving plasmonic states. ${ }^{106}$ The faceted gap geometry may be also an adequate morphology to achieve strong coupling with self-assembled molecules ${ }^{107,108}$ extended over the entire gap. The possibilities of the versatile flat-gap morphology could thus open new venues for simultaneous and independent control of the near-field and far-field responses in nanophotonics.

\section{ASSOCIATED CONTENT}

\section{S Supporting Information}

We provide an additional file where the spectral saturation of the lowest energy mode in the flat-gap antenna is shown, as derived within a circuit theory model. This material is available free of charge via the Internet at http://pubs.acs.org.

\section{AUTHOR INFORMATION}

\section{Corresponding Authors}

*E-mail: r.esteban@ehu.es.

*E-mail: aizpurua@ehu.es.

\section{Notes}

The authors declare no competing financial interest.

\section{ACKNOWLEDGMENTS}

We acknowledge useful discussions with Prof. Jean-Jacques Greffet, Prof. P. Apell, Prof. J. J. Baumberg, and Dr. C. Tzerkezis. J.A. and R.E. acknowledge financial support from the Spanish Ministry of Economy and Competitiveness (project FIS2013-41184-P) and from the Basque Government (project ETORTEK2014-2015). R.E. acknowledges support from the Fellows Gipuzkoa Program of the Gipuzkoako Foru Aldundia. P.N. acknowledges support from the Robert A. Welch Foundation under grant C-1222 and the Office of Naval Research (N00014-10-1-0989). 


\section{REFERENCES}

(1) Mühlschlegel, P.; Eisler, H.-J.; Martin, O. J. F.; Hecht, B.; Pohl, D. W. Resonant optical antennas. Science 2005, 308, 1607-1609.

(2) Novotny, L.; van Hulst, N. Antennas for light. Nat. Photonics 2011, 5, 83-90.

(3) Oldenburg, S. J.; Averitt, R. D.; Westcott, S. L.; Halas, N. J. Nanoengineering of optical resonances. Chem. Phys. Lett. 1998, 288, 243-247.

(4) Pelton, M.; Aizpurua, J.; Bryant, G. W. Metal-nanoparticle plasmonics. Laser Photonics Rev. 2008, 2, 136-159.

(5) Dorfmüller, J.; Vogelgesang, R.; Weitz, R. T.; Rockstuhl, C.; Etrich, C.; Pertsch, T.; Lederer, F.; Kern, K. Fabry-Pérot resonances in one-dimensional plasmonic nanostructures. Nano Lett. 2009, 9, 23722377.

(6) Aubry, A.; Lei, D. Y.; Fernández-Domínguez, A. I.; Sonnefraud, Y.; Maier, S. A.; Pendry, J. B. Plasmonic light-harvesting devices over the whole visible spectrum. Nano Lett. 2010, 10, 2574-2579.

(7) Muskens, O. L.; Giannini, V.; Sánchez-Gil, J. A.; Rivas, J. G. Optical scattering resonances of single and coupled dimer plasmonic nanoantennas. Opt. Express 2007, 15, 17736-17746.

(8) Su, K.-H.; Wei, Q.-H.; Zhang, X.; Mock, J. J.; Smith, D. R.; Schultz, S. Interparticle coupling effects on plasmon resonances of nanogold particles. Nano Lett. 2003, 3, 1087-1090.

(9) Atay, T.; Song, J.-H.; Nurmikko, A. V. Strongly interacting plasmon nanoparticle pairs: from dipole-dipole interaction to conductively coupled regime. Nano Lett. 2004, 4, 1627-1631.

(10) Rechberger, W.; Hohenau, A.; Leitner, A.; Krenn, J. R.; Lamprecht, B.; Aussenegg, F. R. Optical properties of two interacting gold nanoparticles. Opt. Commun. 2003, 220, 137-141.

(11) Tamaru, H.; Kuwata, H.; Miyazaki, H. T.; Miyano, K. Resonant light scattering from individual $\mathrm{Ag}$ nanoparticles and particle pairs. Appl. Phys. Lett. 2002, 80, 1826-1828.

(12) Schuck, P. J.; Fromm, D. P.; Sundaramurthy, A.; Kino, G. S.; Moerner, W. E. Improving the mismatch between light and nanoscale objects with gold bowtie nanoantennas. Phys. Rev. Lett. 2005, 94, 017402 .

(13) Gunnarsson, L.; Rindzevicius, T.; Prikulis, J.; Kasemo, B.; Käll, M.; Zou, S.; Schatz, G. C. Confined plasmons in nanofabricated single silver particle pairs: experimental observations of strong interparticle interactions. J. Phys. Chem. B 2005, 109, 1079-1087.

(14) Jensen, T.; Kelly, L.; Lazarides, A.; Schatz, G. C. Electrodynamics of noble metal nanoparticles and nanoparticle clusters. J. Cluster Sci. 1999, 10, 295-317.

(15) Aizpurua, J.; Bryant, G. W.; Richter, L. J.; García de Abajo, F. J.; Kelley, B. K.; Mallouk, T. Optical properties of coupled metallic nanorods for field-enhanced spectroscopy. Phys. Rev. B 2005, 71, 235420.

(16) Ghenuche, P.; Cherukulappurath, S.; Taminiau, T. H.; van Hulst, N. F.; Quidant, R. Spectroscopic mode mapping of resonant plasmon nanoantennas. Phys. Rev. Lett. 2008, 101, 116805.

(17) Romero, I.; Aizpurua, J.; Bryant, G. W.; García de Abajo, F. J. Plasmons in nearly touching metallic nanoparticles: singular response in the limit of touching dimers. Opt. Express 2006, 14, 9988-9999.

(18) Pérez-González, O.; Zabala, N.; Borisov, A. G.; Halas, N. J.; Nordlander, P.; Aizpurua, J. Optical spectroscopy of conductive junctions in plasmonic cavities. Nano Lett. 2010, 10, 3090-3095.

(19) Large, N.; Abb, M.; Aizpurua, J.; Muskens, O. L. Photoconductively loaded plasmonic nanoantenna as building block for ultracompact optical switches. Nano Lett. 2010, 10, 1741-1746.

(20) Joshi, B. P.; Wei, Q.-H. Cavity resonances of metal-dielectricmetal nanoantennas. Opt. Express 2008, 16, 10315-10322.

(21) Kern, J.; Großmann, S.; Tarakina, N. V.; Häckel, T.; Emmerling, M.; Kamp, M.; Huang, J.-S.; Biagioni, P.; Prangsma, J. C.; Hecht, B. Atomic-scale confinement of resonant optical fields. Nano Lett. 2012, $12,5504-5509$.

(22) Esteban, R.; Teperik, T. V.; Greffet, J. J. Optical patch antennas for single photon emission using surface plasmon resonances. Phys. Rev. Lett. 2010, 104, 026802.
(23) Xu, H.; Bjerneld, E. J.; Käll, M.; Börjesson, L. Spectroscopy of single hemoglobin molecules by surface enhanced Raman scattering. Phys. Rev. Lett. 1999, 83, 4357-4360.

(24) Grillet, N.; Manchon, D.; Bertorelle, F.; Bonnet, C.; Broyer, M.; Cottancin, E.; Lermé, J.; Hillenkamp, M.; Pellarin, M. Plasmon coupling in silver nanocube dimers: resonance splitting induced by edge rounding. ACS Nano 2011, 5, 9450-9462.

(25) Caldwell, J. D.; Glembocki, O. J.; Francescato, Y.; Sharac, N.; Giannini, V.; Bezares, F. J.; Long, J. P.; Owrutsky, J. C.; Vurgaftman, I.; Tischler, J. G.; Wheeler, V. D.; Bassim, N. D.; Shirey, L. M.; Kasica, R.; Maier, S. A. Low-loss, extreme subdiffraction photon confinement via silicon carbide localized surface phonon polariton resonators. Nano Lett. 2013, 13, 3690-3697.

(26) Zuloaga, J.; Prodan, E.; Nordlander, P. Quantum description of the plasmon resonances of a nanoparticle dimer. Nano Lett. 2009, 9, 887-891.

(27) Savage, K. J.; Hawkeye, M. M.; Esteban, R.; Borisov, A. G.; Aizpurua, J.; Baumberg, J. J. Revealing the quantum regime in tunnelling plasmonics. Nature 2012, 491, 574-577.

(28) Scholl, J. A.; García-Etxarri, A.; Koh, A. L.; Dionne, J. A. Observation of quantum tunneling between two plasmonic nanoparticles. Nano Lett. 2013, 13, 564-569.

(29) Tan, S. F.; Wu, L.; Yang, J. K. W.; Bai, P.; Bosman, M.; Nijhuis, C. A. Quantum plasmon resonances controlled by molecular tunnel junctions. Science 2014, 343, 1496-1499.

(30) Kravtsov, V.; Berweger, S.; Atkin, J. M.; Raschke, M. B. Control of plasmon emission and dynamics at the transition from classical to quantum coupling. Nano Lett. 2014, 14, 5270-5275.

(31) Zhu, W.; Crozier, K. B. Quantum mechanical limit to plasmonic enhancement as observed by surface-enhanced Raman scattering. Nat. Commun. 2014, 5, 5228 .

(32) Esteban, R.; Borisov, A. G.; Nordlander, P.; Aizpurua, J. Bridging quantum and classical plasmonics with a quantum-corrected model. Nat. Commun. 2012, 3, 825.

(33) García de Abajo, F. J. Nonlocal effects in the plasmons of strongly interacting nanoparticles, dimers, and waveguides. J. Phys. Chem. C 2008, 112, 17983-17987.

(34) McMahon, J. M.; Gray, S. K.; Schatz, G. C. Optical properties of nanowire dimers with a spatially nonlocal dielectric function. Nano Lett. 2010, 10, 3473-3481.

(35) Ciracì, C.; Hill, R. T.; Mock, J. J.; Urzhumov, Y.; FernándezDomínguez, A. I.; Maier, S. A.; Pendry, J. B.; Chilkoti, A.; Smith, D. R. Probing the ultimate limits of plasmonic enhancement. Science 2012, 337, 1072-1074.

(36) Fernández-Domínguez, A. I.; Wiener, A.; García-Vidal, F. J.; Maier, S. A.; Pendry, J. B. Transformation-optics description of nonlocal effects in plasmonic nanostructures. Phys. Rev. Lett. 2012, $108,106802$.

(37) Toscano, G.; Raza, S.; Jauho, A.-P.; Mortensen, N. A.; Wubs, M. Modified field enhancement and extinction by plasmonic nanowire dimers due to nonlocal response. Opt. Express 2012, 20, 4176-4188.

(38) Luo, Y.; Fernandez-Dominguez, A. I.; Wiener, A.; Maier, S. A.; Pendry, J. B. Surface plasmons and nonlocality: a simple model. Phys. Rev. Lett. 2013, 111, 093901.

(39) Teperik, T. V.; Nordlander, P.; Aizpurua, J.; Borisov, A. G. Quantum effects and nonlocality in strongly coupled plasmonic nanowire dimers. Opt. Express 2013, 21, 27306-27325.

(40) Teperik, T. V.; Nordlander, P.; Aizpurua, J.; Borisov, A. G. Robust subnanometric plasmon ruler by rescaling of the nonlocal optical response. Phys. Rev. Lett. 2013, 110, 263901.

(41) Esteban, R.; Zugarramurdi, A.; Zhang, P.; Nordlander, P.; García-Vidal, F. J.; Borisov, A. G.; Aizpurua, J. A classical treatment of optical tunneling in plasmonic gaps: extending the quantum corrected model to practical situations. Faraday Discuss. DOI: 10.1039/ C4FD00196F.

(42) García de Abajo, F. J.; Howie, A. Retarded field calculation of electron energy loss in inhomogeneous dielectrics. Phys. Rev. B 2002, $65,115418$. 
(43) Xu, H.; Aizpurua, J.; Käll, M.; Apell, P. Electromagnetic contributions to single-molecule sensitivity in surface-enhanced Raman scattering. Phys. Rev. E 2000, 62, 4318-4324.

(44) Nordlander, P.; Oubre, C.; Prodan, E.; Li, K.; Stockman, M. I. Plasmon hybridization in nanoparticle dimers. Nano Lett. 2004, 4, 899-903.

(45) Lebedev, V.; Vergeles, S.; Vorobev, P. Giant enhancement of electric field between two close metallic grains due to plasmonic resonance. Opt. Lett. 2010, 35, 640-642.

(46) Ditlbacher, H.; Hohenau, A.; Wagner, D.; Kreibig, U.; Rogers, M.; Hofer, F.; Aussenegg, F. R.; Krenn, J. R. Silver Nanowires as Surface Plasmon Resonators. Phys. Rev. Lett. 2005, 95, 257403.

(47) Novotny, L. Effective wavelength scaling for optical antennas. Phys. Rev. Lett. 2007, 98, 266802.

(48) Bryant, G. W.; García de Abajo, F. J.; Aizpurua, J. Mapping the plasmon resonances of metallic nanoantennas. Nano Lett. 2008, 8, 631-636.

(49) Dorfmüller, J.; Vogelgesang, R.; Khunsin, W.; Rockstuhl, C.; Etrich, C.; Kern, K. Plasmonic nanowire antennas: experiment, simulation, and theory. Nano Lett. 2010, 10, 3596-3603.

(50) Fischer, H.; Martin, O. J. F. Engineering the optical response of plasmonic nanoantennas. Opt. Express 2008, 16, 9144-9154.

(51) Alù, A.; Engheta, N. Input impedance, nanocircuit loading, and radiation tuning of optical nanoantennas. Phys. Rev. Lett. 2008, 101, 043901.

(52) Alù, A.; Engheta, N. Tuning the scattering response of optical nanoantennas with nanocircuit loads. Nat. Photonics 2008, 2, 307-310.

(53) Locatelli, A.; De Angelis, C.; Modotto, D.; Boscolo, S.; Sacchetto, F.; Midrio, M.; Capobianco, A.-D.; Pigozzo, F. M.; Someda, C. G. Modeling of enhanced field confinement and scattering by optical wire antennas. Opt. Express 2009, 17, 16792-16800.

(54) Yang, J.; Sauvan, C.; Jouanin, A.; Collin, S.; Pelouard, J.-L.; Lalanne, P. Ultrasmall metal-insulator-metal nanoresonators: impact of slow-wave effects on the quality factor. Opt. Express 2012, 20, 1688016891.

(55) Kwon, S.-H. Deep subwavelength plasmonic whispering-gallerymode cavity. Opt. Express 2012, 20, 24918-24924.

(56) Bozhevolnyi, S. I.; Søndergaard, T. General properties of slowplasmon resonant nanostructures: nano-antennas and resonators. Opt. Express 2007, 15, 10869-10877.

(57) Jung, J.; Søndergaard, T.; Bozhevolnyi, S. I. Gap plasmonpolariton nanoresonators: scattering enhancement and launching of surface plasmon polaritons. Phys. Rev. B 2009, 79, 035401.

(58) Kuttge, M.; García de Abajo, F. J.; Polman, A. Ultrasmall mode volume plasmonic nanodisk resonators. Nano Lett. 2010, 10, 15371541.

(59) Lassiter, J. B.; McGuire, F.; Mock, J. J.; Ciracì, C.; Hill, R. T.; Wiley, B. J.; Chilkoti, A.; Smith, D. R. Plasmonic waveguide modes of film-coupled metallic nanocubes. Nano Lett. 2013, 13, 5866-5872.

(60) Minkowski, F.; Wang, F.; Chakrabarty, A.; Wei, Q.-H. Resonant cavity modes of circular plasmonic patch nanoantennas. Appl. Phys. Lett. 2014, 104, 021111.

(61) Gordon, R. Light in a subwavelength slit in a metal: propagation and reflection. Phys. Rev. B 2006, 73, 153405.

(62) Balanis, C. A. Antenna Theory. Analysis and Design; John Wiley \& Sons, Inc.: Hoboken, 2005.

(63) Le, F.; Brandl, D. W.; Urzhumov, Y. A.; Wang, H.; Kundu, J.; Halas, N. J.; Aizpurua, J.; Nordlander, P. Metallic nanoparticle arrays: a common substrate for both surface-enhanced Raman scattering and surface-enhanced infrared absorption. ACS Nano 2008, 2, 707-718.

(64) Miyazaki, H. T.; Kurokawa, Y. Squeezing Visible Light Waves into a 3-nm-Thick and 55-nm-Long Plasmon Cavity. Phys. Rev. Lett. 2006, 96, 097401.

(65) Park, J.; Kim, H.; Lee, I.-M.; Kim, S.; Jung, J.; Lee, B. Resonant tunneling of surface plasmon polariton in the plasmonic nano-cavity. Opt. Express 2008, 16, 16903-16915.

(66) Dionne, J. A.; Sweatlock, L. A.; Atwater, H. A.; Polman, A. Plasmon slot waveguides: towards chip-scale propagation with subwavelength-scale localization. Phys. Rev. B 2006, 73, 035407.
(67) Søndergaard, T.; Beermann, J.; Boltasseva, A.; Bozhevolnyi, S. I. Slow-plasmon resonant-nanostrip antennas: analysis and demonstration. Phys. Rev. B 2008, 77, 115420.

(68) Barnard, E. S.; White, J. S.; Chandran, A.; Brongersma, M. L. Spectral properties of plasmonic resonator antennas. Opt. Express 2008, 16, 16529-16537.

(69) Filter, R.; Qi, J.; Rockstuhl, C.; Lederer, F. Circular optical nanoantennas: an analytical theory. Phys. Rev. B 2012, 85, 125429.

(70) Cirací, C.; Britt Lassiter, J.; Moreau, A.; Smith, D. R. Quasianalytic study of scattering from optical plasmonic patch antennas. J. Appl. Phys. 2013, 114, 163108.

(71) Jacob, Z.; Shalaev, V. M. Plasmonics goes quantum. Science 2011, 334, 463-464.

(72) Tame, M. S.; McEnery, K. R.; Özdemir, S. K.; Lee, J.; Maier, S. A.; Kim, M. S. Quantum plasmonics. Nat. Phys. 2013, 9, 329-340.

(73) Cha, H.; Yoon, J. H.; Yoon, S. Probing quantum plasmon coupling using gold nanoparticle dimers with tunable interparticle distances down to the subnanometer range. ACS Nano 2014, 8, 85548563.

(74) Mao, L.; Li, Z.; Wu, B.; Xu, H. Effects of quantum tunneling in metal nanogap on surface-enhanced Raman scattering. Appl. Phys. Lett. 2009, 94, 243102.

(75) Schnell, M.; García-Etxarri, A.; Huber, A. J.; Crozier, K.; Aizpurua, J.; Hillenbrand, R. Controlling the near-field oscillations of loaded plasmonic nanoantennas. Nat. Photonics 2009, 3, 287-291.

(76) Marinica, D. C.; Kazansky, A. K.; Nordlander, P.; Aizpurua, J.; Borisov, A. G. Quantum plasmonics: nonlinear effects in the field enhancement of a plasmonic nanoparticle dimer. Nano Lett. 2012, 12, 1333-1339.

(77) Mbindyo, J. K. N.; Mallouk, T. E.; Mattzela, J. B.; Kratochvilova, I.; Razavi, B.; Jackson, T. N.; Mayer, T. S. Template synthesis of metal nanowires containing monolayer molecular junctions. J. Am. Chem. Soc. 2002, 124, 4020-4026.

(78) Thomas, K. G.; Barazzouk, S.; Ipe, B. I.; Shibu Joseph, S. T.; Kamat, P. V. Uniaxial plasmon coupling through longitudinal selfassembly of gold nanorods. J. Phys. Chem. B 2004, 108, 13066-13068.

(79) Caswell, K. K.; Wilson, J. N.; Bunz, U. H. F.; Murphy, C. J. Preferential end-to-end assembly of gold nanorods by biotinstreptavidin connectors. J. Am. Chem. Soc. 2003, 125, 13914-13915.

(80) Kim, D.-S.; Heo, J.; Ahn, S.-H.; Han, S. W.; Yun, W. S.; Kim, Z. $\mathrm{H}$. Real-space mapping of the strongly coupled plasmons of nanoparticle dimers. Nano Lett. 2009, 9, 3619-3625.

(81) Funston, A. M.; Novo, C.; Davis, T. J.; Mulvaney, P. Plasmon coupling of gold nanorods at short distances and in different geometries. Nano Lett. 2009, 9, 1651-1658.

(82) Jones, S. T.; Taylor, R. W.; Esteban, R.; Abo-Hamed, E. K.; Bomans, P. H. H.; Sommerdijk, N. A. J. M.; Aizpurua, J.; Baumberg, J. J.; Scherman, O. A. Gold nanorods with sub-nanometer separation using cucurbit[n]uril for SERS applications. Small 2014, 10, 42984303.

(83) Joshi, B.; Wen, X.; Sun, K.; Lu, W.; Wei, Q.-H. Fabrication and characterization of coupled metal-dielectric-metal nanoantennas. J. Vac. Sci. Technol. B 2010, 28, C6O21-C6O25.

(84) Alaee, R.; Menzel, C.; Huebner, U.; Pshenay-Severin, E.; Bin Hasan, S.; Pertsch, T.; Rockstuhl, C.; Lederer, F. Deep-subwavelength plasmonic nanoresonators exploiting extreme coupling. Nano Lett. 2013, 13, 3482-3486.

(85) Sigle, D. O.; Mertens, J.; Herrmann, L. O.; Bowman, R. W.; Ithurria, S.; Dubertret, B.; Shi, Y.; Yang, H. Y.; Tserkezis, C.; Aizpurua, J.; Baumberg, J. J. Monitoring morphological changes in 2D monolayer semiconductors using atom-thick plasmonic nanocavities. ACS Nano $10.1021 / \mathrm{nn} 5064198$

(86) Rockstuhl, C.; Salt, M. G.; Herzig, H. P. Analysis of the phononpolariton response of silicon carbide microparticles and nanoparticles by use of the boundary element method. J. Opt. Soc. Am. B 2005, 22, 481-487.

(87) Ameen, M.; Garcia-Etxarri, A.; Schnell, M.; Hillenbrand, R.; Aizpurua, J. Infrared phononic nanoantennas: localized surface phonon polaritons in SiC disks. Chin. Sci. Bull. 2010, 55, 2625-2628. 
(88) Esteban, R.; Aizpurua, J.; Bryant, G. W. Strong coupling of single emitters interacting with phononic infrared antennae. New J. Phys. 2014, 16, 013052.

(89) Palik, E. Handbook of Optical Constants of Solids; Academic Press: San Diego, 1985.

(90) Mutschke, H.; Andersen, A. C.; Clément, D.; Henning, Th.; Peiter, G. Infrared properties of SiC particles. Astron. Astrophys. 1999, 345, 187-202.

(91) Spitzer, W. G.; Kleinman, D.; Walsh, D. Infrared properties of hexagonal silicon carbide. Phys. Rev. 1959, 113, 127-132.

(92) Le Gall, J.; Olivier, M.; Greffet, J.-J. Experimental and theoretical study of reflection and coherent thermal emission by a $\mathrm{SiC}$ grating supporting a surface-phonon polariton. Phys. Rev. B 1997, 55, 1010510114.

(93) Chalopin, Y.; Dammak, H.; Hayoun, M.; Besbes, M.; Greffet, J.J. Size-dependent infrared properties of $\mathrm{MgO}$ nanoparticles with evidence of screening effect. Appl. Phys. Lett. 2012, 100, 241904.

(94) Chang, Y.-C.; Wang, S.-M.; Chung, H.-C.; Tseng, C.-B.; Chang, S.-H. Observation of absorption-dominated bonding dark plasmon mode from metal-insulator-metal nanodisk arrays fabricated by nanospherical-lens lithography. ACS Nano 2012, 6, 3390-3396.

(95) Zhang, R.; Zhang, Y.; Dong, Z. C.; Jiang, S.; Zhang, C.; Chen, L. G.; Zhang, L.; Liao, Y.; Aizpurua, J.; Luo, Y.; Yang, J. L.; Hou, J. G. Chemical mapping of a single molecule by plasmon-enhanced Raman scattering. Nature 2013, 498, 82-86.

(96) Hartschuh, A.; Sánchez, E.; Xie, X.; Novotny, L. High-resolution near-field Raman microscopy of single-walled carbon nanotubes. Phys. Rev. Lett. 2003, 90, 095503.

(97) Deckert-Gaudig, T.; Deckert, V. Ultraflat transparent gold nanoplates-ideal substrates for tip-enhanced Raman scattering experiments. Small 2009, 5, 432-436.

(98) García-Etxarri, A.; Romero, I.; García de Abajo, F. J.; Hillenbrand, R.; Aizpurua, J. Influence of the tip in near-field imaging of nanoparticle plasmonic modes: weak and strong coupling regimes. Phys. Rev. B 2009, 79, 125439.

(99) Pettinger, B.; Schambach, P.; Villagómez, C. J.; Scott, N. Tipenhanced Raman spectroscopy: near-fields acting on a few molecules. Annu. Rev. Phys. Chem. 2012, 63, 379-399.

(100) Stockman, M. I.; Kling, M. F.; Kleineberg, U.; Krausz, F. Attosecond nanoplasmonic-field microscope. Nat. Photonics 2007, 1, 539-544.

(101) Dombi, P.; Hörl, A.; Rácz, P.; Márton, I.; Trügler, A.; Krenn, J. R.; Hohenester, U. Ultrafast strong-field photoemission from plasmonic nanoparticles. Nano Lett. 2013, 13, 674-678.

(102) Danckwerts, M.; Novotny, L. Optical frequency mixing at coupled gold nanoparticles. Phys. Rev. Lett. 2007, 98, 026104.

(103) Benz, F.; Tserkezis, C.; Herrmann, L. O.; de Nijs, B.; Sanders, A.; Sigle, D. O.; Pukenas, L.; Evans, S. D.; Aizpurua, J.; Baumberg, J. J. Nanooptics of molecular-shunted plasmonic nanojunctions. Nano Lett. $10.1021 / \mathrm{nl} 5041786$

(104) Govorov, A. O.; Lee, J.; Kotov, N. A. Theory of plasmonenhanced Förster energy transfer in optically excited semiconductor and metal nanoparticles. Phys. Rev. B 2007, 76, 125308.

(105) Durach, M.; Rusina, A.; Klimov, V. I.; Stockman, M. I. Nanoplasmonic renormalization and enhancement of Coulomb interactions. New J. Phys. 2008, 10, 105011.

(106) Gonzalez-Tudela, A.; Martin-Cano, D.; Moreno, E.; MartinMoreno, L.; Tejedor, C.; Garcia-Vidal, F. J. Entanglement of two qubits mediated by one-dimensional plasmonic waveguides. Phys. Rev. Lett. 2011, 106, 020501.

(107) Bellessa, J.; Bonnand, C.; Plenet, J.; Mugnier, J. Strong coupling between surface plasmons and excitons in an organic semiconductor. Phys. Rev. Lett. 2004, 93, 036404.

(108) Savasta, S.; Saija, R.; Ridolfo, A.; Di Stefano, O.; Denti, P.; Borghese, F. Nanopolaritons: vacuum Rabi splitting with a single quantum dot in the center of a dimer nanoantenna. ACS Nano 2010, 4, 6369-6376. 